\section{The Consequence of the Relationship between Shoulder and Cervical Multifiduc Muscles Contractions}

Received: April 04, 2017; Accepted: April 21, 2017; Published: April 25, 2017

\section{Asghar Rezasoltani and Shahid Beheshti \\ University of Medical Sciences,} Physiotherapy Research Centre, School of Rehabilitation, Tehran, Iran

Corresponding author: Asghar Rezasoltani

Đ a_rezasoltani@sbmu.ac.ir

University of Medical Sciences, Physiotherapy Research Centre, School of Rehabilitation, Tehran, Iran.

Citation: Rezasoltani A, Beheshti S. The Consequence of the Relationship between Shoulder and Cervical Multifiduc Muscles Contractions. Spine Res. 2017, 3:1.

contraction increased in all directions of movements. Further, the authors imply that the MM was more reactive in higher levels of shoulder muscle contraction and more in shoulder abductor and external rotator than the other directions [6].

This work well advances our knowledge of the relationship between $\mathrm{MM}$ and shoulder muscle contraction in healthy individuals. The main overall outcome of this study would be to apply the method of testing in a form of training to improve the function of MM in a group of patients with neck pain. The method of this kind of training has been already applied in healthy subjects where the dimension of cervical MM was reported to be increased after 12 weeks of shoulder abductor muscle trainings [7]. Therefore, to strengthen the neck stabilizer muscles, it may be possible to employ shoulder muscle performance where the neck movement is painful or prohibited. The method is also applicable in those individuals who are at risk of developing neck pain e.g. office workers and dentists by means of prevention. An easy way of doing this kind of exercise is recommended by using a suitable tra-band and performing isotonic shoulder muscle contractions (Figures 1a and 1b). Three sets of isotonic shoulder abduction a day with ten times per each set and one minute rest interval between each set is recommended. 

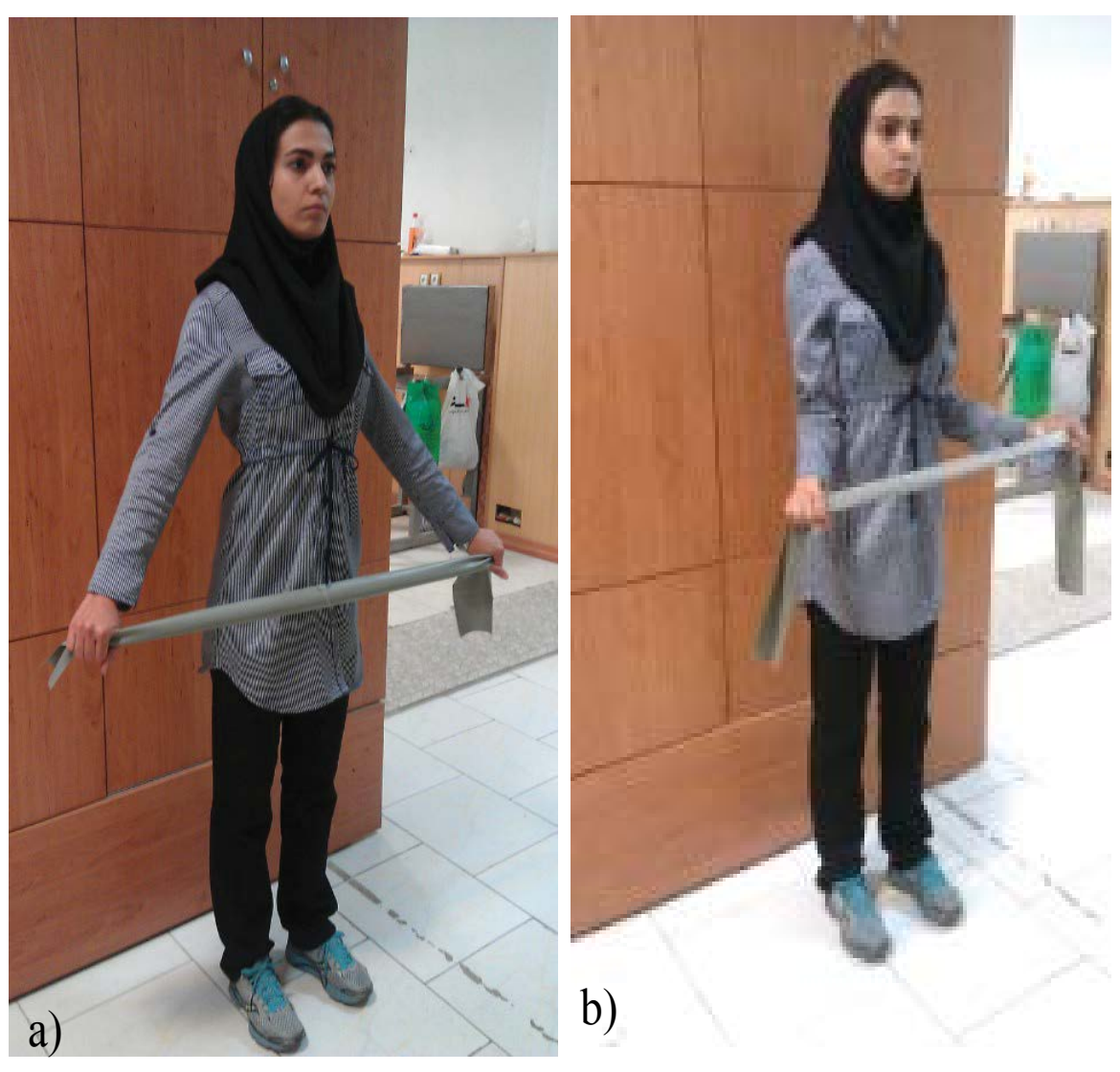

Figure 1 1a, and $\mathbf{1 b}$ Isotonic shoulder abductors (a) and external rotators (b) muscles training by using a tra-band. 


\section{References}

1 Anderson JS, Hsu AW, Vasavada AN (2005) Morphology, architecture, and biomechanics of human cervical multifidus. Spine 30: 86-91.

2 Ward SR, Kim CW, Eng CM, Ghottschalk LJ, Tomyia A (2009) Architectural analysis and intraoperative mesurements demonstrate the unique design of the multifidus muscle for lumbar spine stability. J Bone Joint Surg 91: 176-185.

3 Nuzzo JL, McCaulley GO, Cormie P, Cavill MG, McBride JM (2008) Trunk muscle activity during stability ball and free weight exercises. J Strength Condition Res 1: 95-102.

4 Danneels LA, Vanderstraeten GG, Cambier DC, Witvrouw EE, Borguis $\mathrm{J}$, et al. (2001) Effects of three different training modalities on the cross sectional area of the lumbar multifidus muscle in patients with chronic low back pain. Br J Sports Med 35: 186-191.

5 Rahnama L, Rezasoltani A, Khalkhali Zavieh M, Noori Kochi F, Akbarzadeh Baghban A (2014) The effects of isometric contraction of shoulder muscles on cervical multifidus muscle dimensions in healthy office workers. J Bodyw Mov Ther 18: 383-389.

6 Rahnama L, Rezasoltani A, Khalkhali Zavieh M, NooriKochi F, Akbarzadeh Baghban A (2015) Differences in cervical multifidus muscle thickness during isometric contraction of shoulder muscles: A comparison between patients with chronic neck pain and healthy controls. J Manipulative Physiol Ther 3: 210-216.

7 Mohammadi M, Rezasoltani A, Khalkhalizavieh M, Tabatabaee SM (2013) A study on the effectiveness of shoulder strengthening exercise on the thickness of neck extensor muscles. J Rehab Med 2: 7-13. 